\section{A patient with bronchiectasis present- ed with progressive dyspnoea and an unresolved right lower lung infiltrate}

\section{CLINICAL PRESENTATION}

A 71-year-old female non-smoker had bronchiectasis with productive cough for decades. Two months prior to this admission, she developed dyspnoea on exertion with more severe cough than before. There was no associated fever, night sweats, poor appetite or weight loss. Chest radiography displayed diffuse bronchiectatic changes on the bilateral airways, with multiple ill defined infiltrates and a large right lower lung patch containing multiple cystic lesions (fig 1). She was initially treated as having community acquired pneumonia but the symptoms persisted.

On admission, the patient was afebrile, conscious and coherent. Physical and neurological examinations were unremarkable except for decreased breath sounds with localised inspiratory crackles and expiratory wheezes on the right lower lung field. Biochemistry studies were within normal limits, while the haemogram showed mild anaemia (haemoglobin: $11.2 \mathrm{~g} / \mathrm{dl}$ ). The serology test for HIV was negative. Sputum cultures yielded Haemophilus influenza but acid fast bacilli were negative on smears. Pulmonary function test showed a severe mixed obstructive and restrictive ventilatory defect.

Chest CT demonstrated bilateral bronchiectasis and a large mass lesion on the right lower lung field, interspersed with multiple parenchymal necrosis and subpleural patches with air bronchograms on the outer layer of the mass (fig 2A, B). There were also multiple mediastinal and hilar lymphadenopathies, with central necrosis and ring enhancement (fig 2C). A chest ultrasound revealed a right lower lung mass composed of solid parts interspersed with air bronchograms and cystic components filled with dense fluid, while a Doppler study showed hypervascularity within the lesion. Bronchoscopy disclosed profuse purulent sputum over the bilateral distal bronchial trees, but without endobronchial lesions.

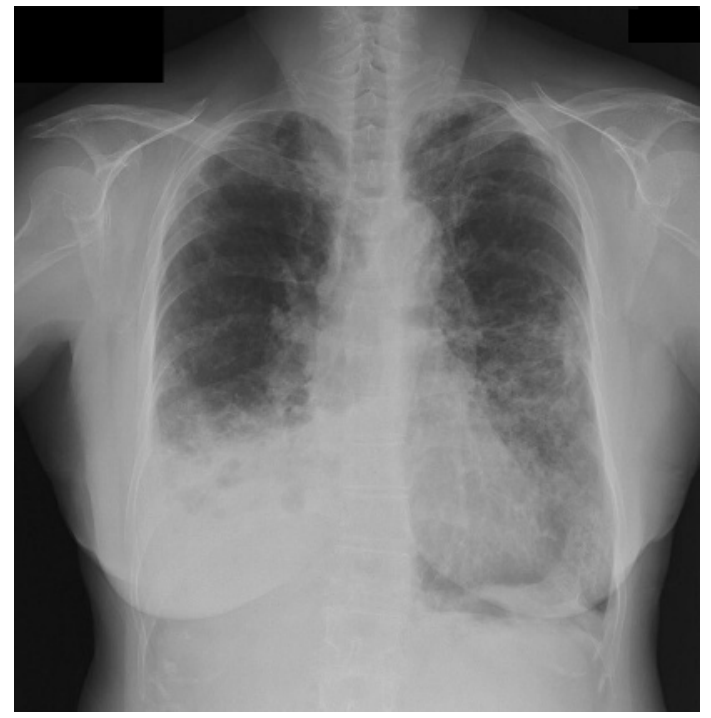

Figure 1 Chest radiograph showing bronchiectasis and a massive right lower lung patch with cystic lesions.
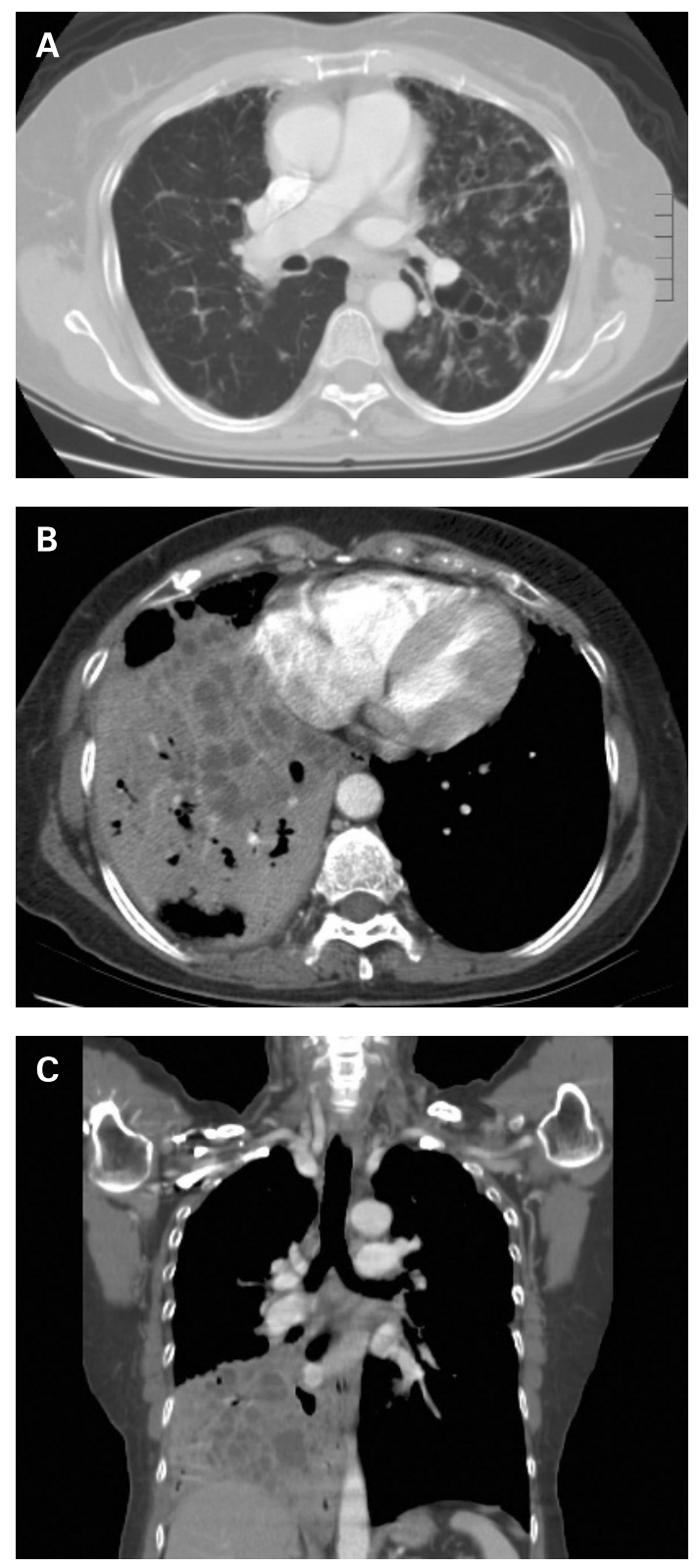

Figure 2 Chest CT scan (A) disclosed bilateral bronchiectasis and $(B, C)$ a mass on the right lower lung with parenchymal necrosis and multiple subpleural patches. Mediastinal and hilar lymphadenopathies were also noted.

\section{QUESTION}

What is your tentative diagnosis?

See page 932 for answer

This case is submitted by:

\section{P-Y Lin, ${ }^{1}$ C-J Yu, ${ }^{1}$ M Yao, ${ }^{1}$ S-C Ku, ${ }^{1}$ L-N Lee, ${ }^{1,2}$ P-C Yang ${ }^{1}$}

1 Department of Internal Medicine, National Taiwan University Hospital, Taipei, Taiwan; ${ }^{2}$ Department of Laboratory Medicine, National Taiwan University Hospital, Taipei, Taiwan

Correspondence to: Dr S-C Ku, Division of Chest Medicine, Department of Internal Medicine, National Taiwan University Hospital, \#7, Chung-Shan South Road, Taipei 100, Taiwan; scku1015@ntu.edu.tw

\section{Competing interests: None.}

Patient consent: Obtained.

Thorax 2008;63:876. doi:10.1136/thx.2007.091736 